\title{
A Novel Approach to Density Near-Zero Acoustic Metamaterials
}

\author{
Milan Sečujski, Norbert Cselyuszka, and Vesna Crnojevićc-Bengin \\ Department of Power Engineering, Electronics and Telecommunications, Faculty of Technical Sciences, University of Novi Sad, \\ 21000 Novi Sad, Serbia \\ Correspondence should be addressed to Milan Sečujski; secujski@uns.ac.rs
}

Received 15 May 2015; Accepted 13 September 2015

Academic Editor: Jainagesh A. Sekhar

Copyright ( 2015 Milan Sečujski et al. This is an open access article distributed under the Creative Commons Attribution License, which permits unrestricted use, distribution, and reproduction in any medium, provided the original work is properly cited.

\begin{abstract}
The study demonstrates the possibility of achieving near-zero propagation of sound waves in acoustic metamaterials based on a membrane-based metamaterial unit cell which exhibits effective mass density of Lorentzian type. The unit cell, which represents the acoustic counterpart of the split ring resonator, was previously used as a building block of left-handed metamaterials, as it exhibits negative density at certain frequencies. In this study we show that its application can be extended to achieving propagation of sound waves at a frequency where its effective density equals zero. This effect can be exploited in a range of applications where extremely low phase variation over long physical distances is required, such as energy tunneling or tailoring the acoustic radiation phase pattern in arbitrary ways. After discussing the dependence of the frequency response of the unit cell on the properties of the host, we show that it can be used to design near-zero acoustic filters with low insertion loss and steep roll-off. Finally, we show that it can be used to achieve simultaneous near-zero propagation at multiple, independently chosen frequencies.
\end{abstract}

\section{Introduction}

Acoustic metamaterials are artificially designed mechanical structures used to control the propagation of sound waves in ways generally not possible in conventional media. They typically include periodic inhomogeneities whose dimensions are sufficiently small in comparison to the wavelength, allowing the material to be treated as homogenous and described by its effective parameters. As opposed to electromagnetic (EM) metamaterials, where specific design is adopted to manipulate the values of effective electric permittivity or magnetic permeability, the research in the field of acoustic metamaterials has principally focused on obtaining metamaterials with unconventional values of compressibility $\beta$ or mass density $\rho[1-7]$.

In our previous research [8], we proposed a novel acoustic metamaterial unit cell based on the analogy with the split ring resonator (SRR), an element regularly used to obtain negative magnetic permeability in EM metamaterials. The proposed acoustic metamaterial unit cell (acoustic split ring resonator, aSRR) consisted of a thin elastic membrane placed in a short tube coaxial with an acoustic duct, as shown in Figure 1.
Its mechanical layout was defined so as to match its lumpedelement equivalent electrical circuit to the one of a typical resonant-permeability EM unit cell consisting of an SRRloaded microstrip, given in [9]. The effective density of the proposed unit cell was shown to be equal to

$$
\rho_{\mathrm{eff}}=\frac{A_{e}+A_{i}}{\left(1 / \rho_{0}\right) A_{e}+\left(1 /\left(\rho^{\prime}-8 \pi \tau / \omega^{2} A_{i} d\right)\right) A_{i}},
$$

where $A_{i}=r^{2} \pi$ is the cross-sectional area of the tube (internal duct), $A_{e}=\left(R^{2}-r^{2}\right) \pi$ is the remaining crosssectional area of the duct external to the tube, $d$ is tube length, $\tau$ is membrane tension, $\rho_{0}$ is air density, and $\rho^{\prime}=$ $\rho_{0}\left(1+M / \rho_{0} A_{i} d\right)$ is the average density in the tube, where $M$ is the mass of the membrane [8]. Unlike other membranebased approaches to achieving effective mass density which is not always positive [4-7], where effective density exhibits Drude type behaviour, the proposed approach resulted in an effective density of the Lorentz type. In this paper we show that the same unit cell, with an appropriate choice of host medium, can be used to achieve near-zero (NZ) acoustic wave propagation at either of the two frequencies where its effective density, given by (1), is equal to zero. 


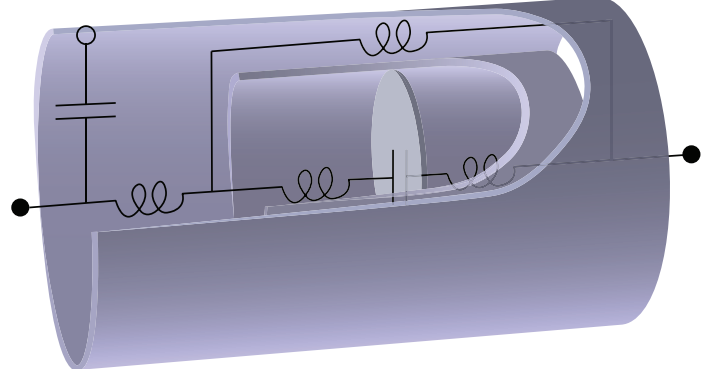

(a)

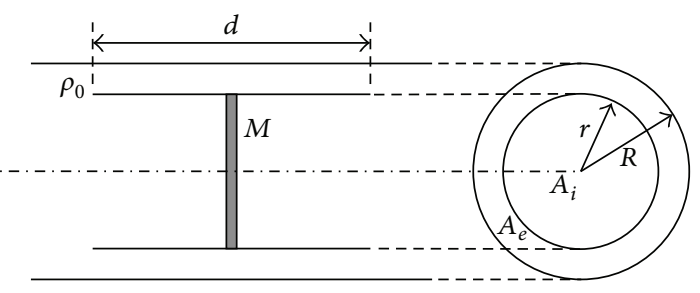

(b)

FIgURE 1: Acoustic split ring resonator (aSRR). (a) Layout and equivalent circuit. (b) Cross-section.

\section{Effective Density from Transmission Line Model and Simulation}

In order to validate the analytically derived expression for effective density given by (1), the same parameter has been extracted from a transmission line (TL) model as well as a finite element method (FEM) simulation in COMSOL Multiphysics using the approach analogous to the one developed for EM metamaterials $[10,11]$. The values of the parameters used in both approaches were $M=0.88 \mathrm{mg}, d=18 \mathrm{~mm}, R=$ $4 \mathrm{~mm}, r=3 \mathrm{~mm}$, and $\tau=5 \mathrm{~N} / \mathrm{m}$, and the resulting resonant frequency was $f_{r}=1175 \mathrm{~Hz}$.

In the approach based on TL modeling, each acoustically short section of the aSRR is modeled with series impedance $Z$ and shunt admittance $Y$ or, alternatively, as a two-port network with the transfer matrix given by

$$
\left[\begin{array}{l}
p_{i} \\
v_{i}
\end{array}\right]=\left[\begin{array}{cc}
\cosh (\gamma l) & Z_{0} \sinh (\gamma l) \\
Z_{0}^{-1} \sinh (\gamma l) & \cosh (\gamma l)
\end{array}\right]\left[\begin{array}{c}
p_{o} \\
v_{o}
\end{array}\right],
$$

where $p_{i}, p_{o}, v_{i}$, and $v_{o}$ are input and output pressures and velocities, $l$ is the length of the section, $Z_{0}=\sqrt{Z / Y}$ is its characteristic impedance, and $\gamma=\sqrt{Z Y}$ is the propagation constant. The losses due to air viscosity and thermal loss are negligible, but the narrow pipe effect has to be taken into account [12-14]. The series impedance and the shunt admittance in this case are given by

$$
\begin{aligned}
& Z=j\left(\frac{\omega \rho_{0}}{A}\right)\left(1-F_{v}\right)^{-1}, \\
& Y=j\left(\frac{A \omega}{\rho_{0} c^{2}}\right)\left(1+(\gamma-1) F_{t}\right),
\end{aligned}
$$

where $A$ is the relevant cross-section area, $\omega$ is the angular frequency, $\rho_{0}$ is the density of air, $c$ is the sound velocity in air, while $F_{v}$ and $F_{t}$ are given by

$$
\begin{aligned}
& F_{v}=\frac{2}{r_{v} \sqrt{-j}} \frac{J_{1}\left(r_{v} \sqrt{-j}\right)}{J_{0}\left(r_{v} \sqrt{-j}\right)}, \\
& F_{t}=\frac{2}{r_{t} \sqrt{-j}} \frac{J_{1}\left(r_{t} \sqrt{-j}\right)}{J_{0}\left(r_{t} \sqrt{-j}\right)} .
\end{aligned}
$$

In (4) $J_{m}$ is the Bessel function of the first kind, order $m$, and $r_{v}$ and $r_{t}$ are given by

$$
\begin{aligned}
& r_{v}=r \sqrt{\frac{\rho \omega}{\mu}}, \\
& r_{t}=r_{v} \sqrt{\frac{\mu C_{p}}{\lambda}}=\sigma r_{v},
\end{aligned}
$$

where $C_{p}$ is specific thermal capacity at a constant pressure, $\lambda$ is thermal conductivity, $\mu$ is shear viscosity, and $\sigma$ is the square root of the Prandtl number. The thin membrane is modelled as the impedance:

$$
Z_{M}=-\frac{j \omega m}{S^{2}} \frac{I_{1}\left(k_{m} r\right) J_{0}\left(k_{m} r\right)+J_{1}\left(k_{m} r\right) I_{0}\left(k_{m} r\right)}{I_{1}\left(k_{m} r\right) J_{2}\left(k_{m} r\right)-J_{1}\left(k_{m} r\right) I_{2}\left(k_{m} r\right)},
$$

where $r$ is the membrane radius, $J_{n}$ and $I_{n}$ are the regular and the modified Bessel function of $n$th order, $m$ is the mass of the membrane, $S$ its surface area, while $k_{m}$ is the wave number obtained from flexural wave equation:

$$
k_{m}^{2}=\omega \sqrt{\frac{\rho_{m}^{\prime \prime}}{D}} .
$$

In (7) $\rho_{m}^{\prime \prime}$ is the surface mass density of the membrane and $D$ is its flexural rigidity, equal to

$$
D=\frac{E h^{3}}{12\left(1-v^{2}\right)},
$$

where $E$ is Young's modulus, $h$ is the thickness of the membrane, and $v$ is Poisson's coefficient. In our TL model and FEM simulations we adopted the values given for DuPont Kapton FPC in COMSOL Multiphysics $\left(E=2.758 \mathrm{GPa}, \rho_{m}=\right.$ $\left.1420 \mathrm{~kg} / \mathrm{m}^{3}, v=0.34\right)$, while the thickness of the membrane was set to $h=22 \mu \mathrm{m}$, yielding the previously specified value of membrane mass $M$.

Figure 2(a) shows a comparison between the effective density obtained from (1) and the same parameter obtained from TL model and FEM simulation. A good match between the results obtained from analytical expression, the model and the simulation can be seen, having in mind that the difference in the magnitudes can be attributed to the fact that (1) refers to the lossless case. 


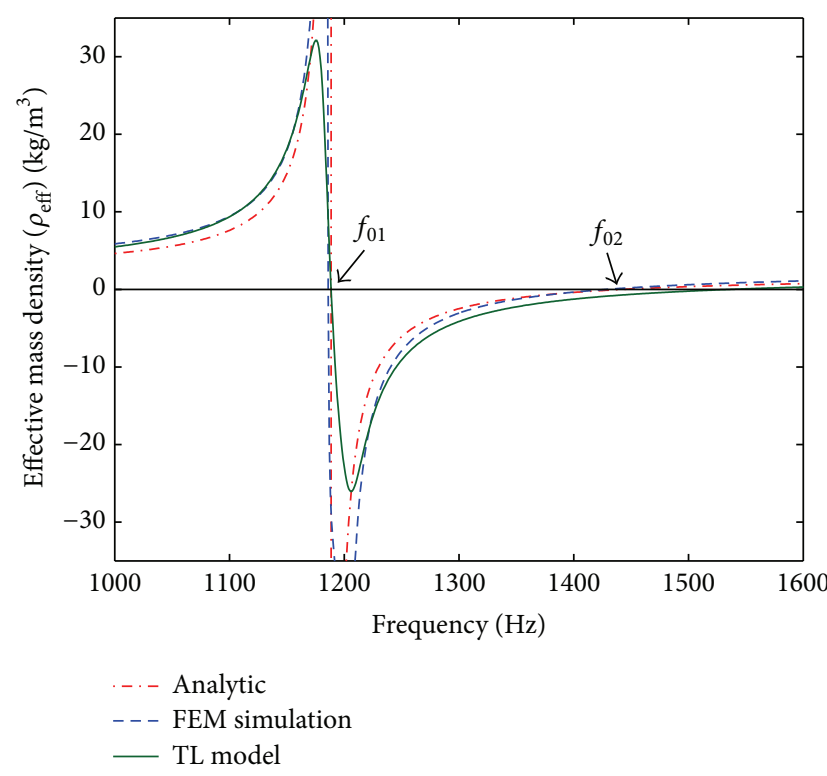

(a)

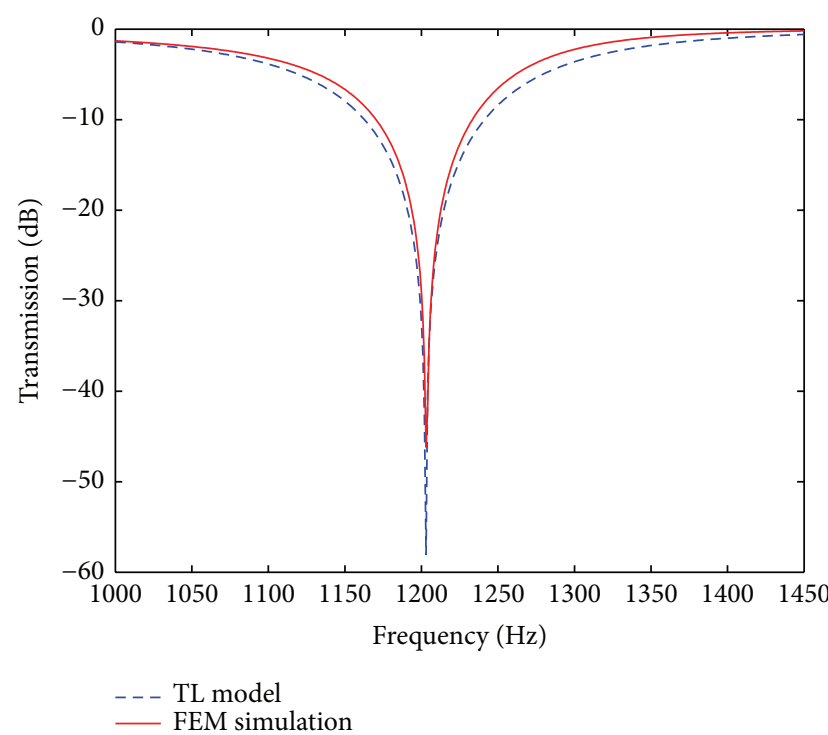

(b)

FIGURE 2: (a) Effective mass density of the aSRR obtained from the analytical expression, TL model, and FEM simulation. (b) Transmission coefficient obtained from TL model and FEM simulation.

\section{Near-Zero Wave Propagation}

Effective density as a function of frequency exhibits resonant behaviour, and there are two frequencies, $f_{01}<f_{r}$ and $f_{02}>$ $f_{r}$, at which its value is equal to zero. From the simulated transmission coefficient, shown in Figure 2(b), it can be seen that the frequency response of the aSRR exhibits a notch around $f_{r}$; that is, the structure behaves as a single-negative metamaterial and does not support propagating waves. For that reason, near-zero propagation at $f_{01}$ is characterized by very high attenuation. At $f_{02}$ attenuation is significantly lower, but the near-zero character of the propagation at $f_{02}$ is not obvious because it is not easily distinguishable from conventional propagation at frequencies immediately above. It can be concluded that, for the purpose of clearly demonstrating near-zero propagation, an all-pass acoustic duct is not a convenient host medium. A convenient host should exhibit a stopband at the frequency range of interest and it should not support double negative (left-handed) wave propagation. In this specific case, where effective density can have both positive and negative values, this implies that the effective compressibility $\beta_{\text {eff }}$ should be strictly positive. An example of such a host is a simple acoustic low-pass filter, either CLC or LCL, provided that the aSRR operates above its cut-off frequency.

In order to demonstrate density near-zero (DNZ) wave propagation we opted for a CLC filter as a host and loaded its inductive section with one aSRR, as shown in Figure 3(a) (not to scale). A TL model was created, similar to the one described in the previous section, and a corresponding FEM simulation was also carried out in COMSOL Multiphysics. The resulting transmission coefficient is shown in Figure 3(b), where a very good match between the TL model and
FEM simulation can be seen. The dashed dotted green line indicates the frequency response of the host low-pass filter. The transmission peak in the stopband of the host corresponds to one of the frequencies where effective density is equal to zero, namely, to $f_{02}$. The frequency response exhibits a transmission zero as well, and its frequency corresponds to the notch in the frequency response of the aSRR itself. It should be noted that in this case the frequency of the transmission peak is higher than the frequency of the transmission zero.

It is also possible to demonstrate $\mathrm{DNZ}$ wave propagation by loading each of the two capacitive sections of the CLC host with an aSRR, as shown in Figure 4(a). A transmission peak and a transmission zero appear in the stopband of the host filter again, their frequencies corresponding to the zero value of the effective density and to the notch in the frequency response of the aSRR, respectively. However, in this case, the frequency of the transmission peak corresponds to $f_{01}$, and it is lower than the frequency of the transmission zero, as shown in Figure 4(b).

It should be noted that when the unit cell is placed in a host structure, its effective density changes because the space between the unit cell and the wall of the host acts like an additional external duct. This includes an additional parallel inductance in the equivalent circuit and eventually results in a shift of the resonant frequency of the aSRR, visible in the insets of both Figures 3(b) and 4(b). Consequently, the actual values of $f_{01}$ and $f_{02}$ are also different than those indicated in Figure 2(b). The shift in the case of the CLC host is more noticeable because the inductance of the additional external duct is significantly smaller than the inductance of the actual external duct of the aSRR. 


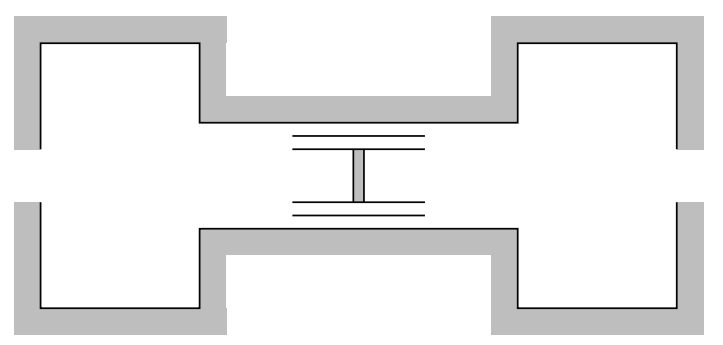

(a)

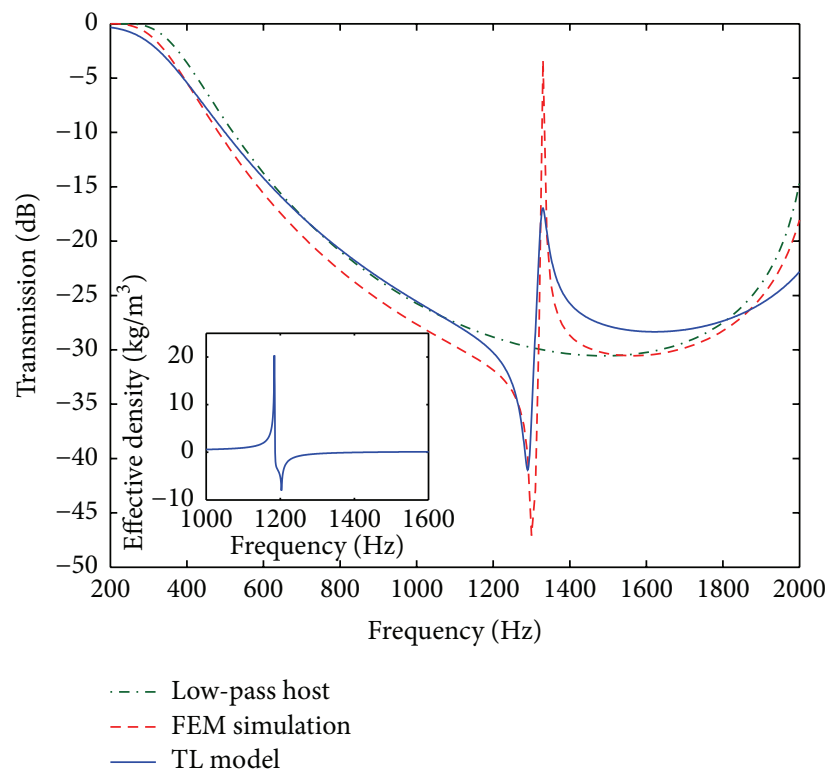

(b)

FIGURE 3: Low-pass acoustic CLC filter (C sections $\varnothing 52 \times 20 \mathrm{~mm}$, L section $\varnothing 10 \times 80 \mathrm{~mm}$ ) loaded with one aSRR in the $\mathrm{L}$ section. (a) Layout. (b) Frequency response obtained through TL model and FEM simulation.

It can also be shown that the situation does not change significantly if both inductive sections of an LCL low-pass host or just the single inductive section of a CLC low-pass host are loaded with an aSRR. Whether the transmission peak will be at a higher frequency than the transmission zero or vice versa depends only on whether the aSRR is located in the inductive or the capacitive section of the host filter.

\section{Near-Zero Bandpass Filtering}

The versatility of the acoustic split ring resonator will be illustrated with an example of its use in NZ bandpass filtering. Namely, it has been shown that in case aSRR is located in the inductive section of the host filter, the transmission peak is at a higher frequency than the transmission zero, while in case it is located in the capacitive section of the host filter, the opposite holds. Let us suppose that all three sections of a CLC host are loaded with one aSRR each, as shown in Figure 5(a), but that the dimensions of the aSRRs are slightly different, chosen so as to set their transmission peaks at the same frequency. The aSRR in the inductive section is expected to introduce a transmission zero at a frequency immediately

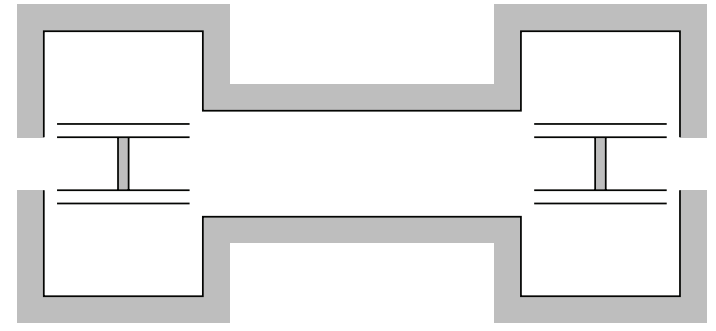

(a)

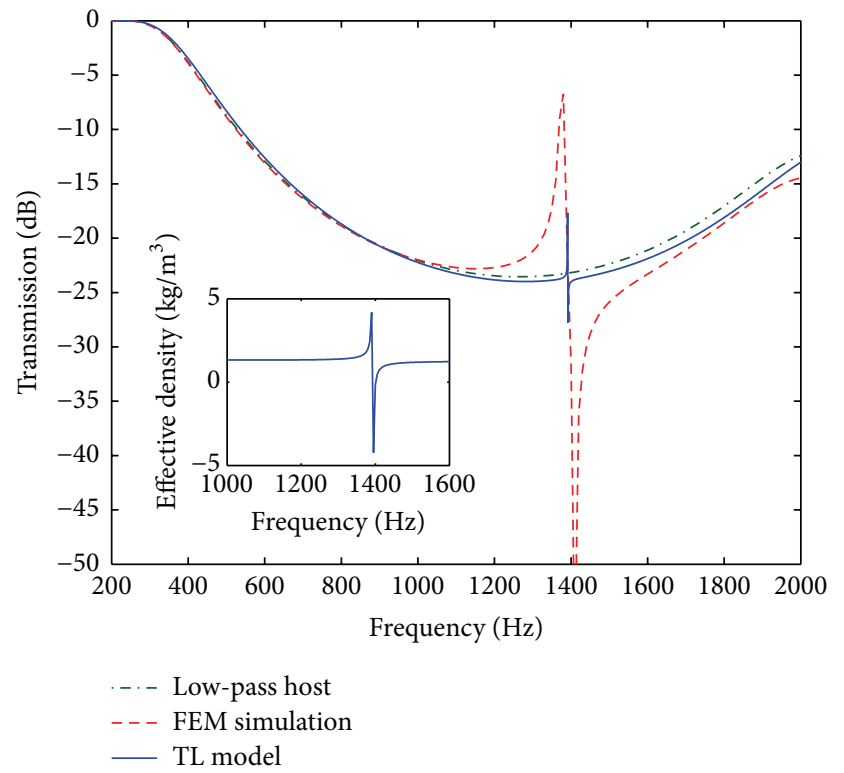

(b)

FIGURE 4: (a) Low-pass acoustic CLC filter loaded with two aSRRs in its capacitive sections. (a) Layout. (b) Frequency response.

below the transmission peak, while the aSRRs in the capacitive sections are expected to introduce transmission zeros at a frequency immediately above. The frequency response of the entire structure will thus correspond to a bandpass filter with very small fractional bandwidth and a steep roll-off. This expectation has been confirmed by a FEM simulation, whose results are shown in Figure 5(b). The bandwidth of the obtained filter is equal to $30 \mathrm{~Hz}$ (corresponding to a fractional bandwidth of $2.4 \%$ ), while the insertion loss is as low as $0.2 \mathrm{~dB}$.

It should be noted that, unlike left-handed structures, NZ filters do not suffer from inherently high passband insertion losses. Namely, from Figure 2(b), it can be seen that the value of the transmission coefficient at the near-zero frequency is relatively high (i.e., the losses are relatively low). On the other hand, it exhibits significantly lower values in the frequency range where the effective density is negative, that is, at frequencies where double negative devices operate, which results in high insertion losses.

Previous research [9] suggests that the mechanisms for reducing insertion loss, increasing the fractional bandwidth or achieving multiband propagation, can be easily extended to the case of DNZ propagation as well. For example, a dualband aSRR, shown in Figure 6(a), has been shown to exhibit 


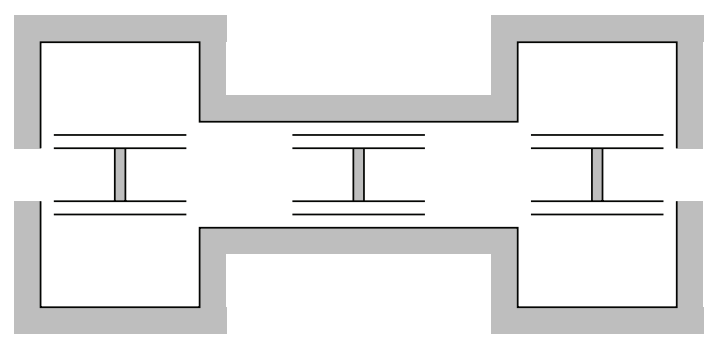

(a)

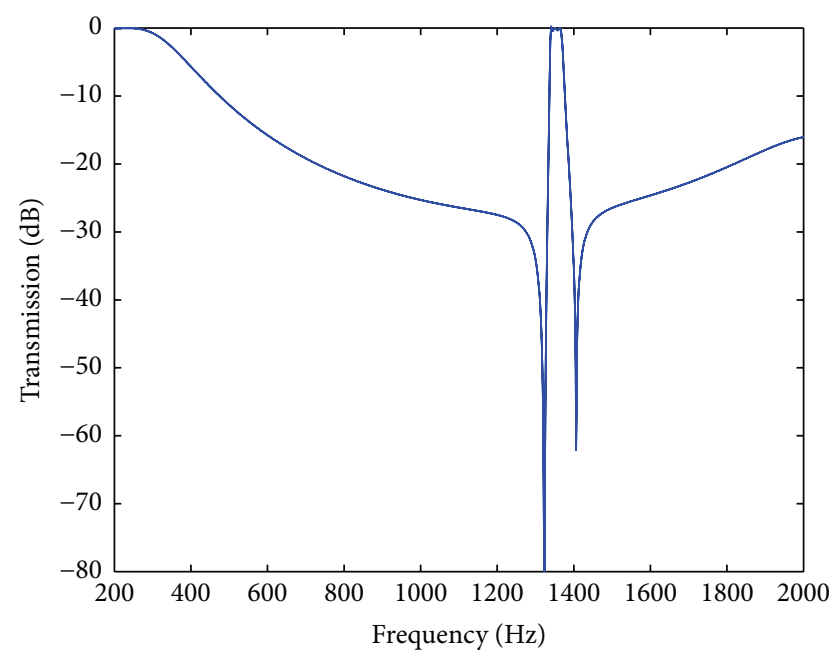

(b)

FIgURE 5: Near-zero bandpass filter. (a) Layout (dimensions are the same as in the previous case except for $d_{1}=18.95 \mathrm{~mm}$ for the aSRR in the inductive section of the host, and $d_{2}=17.4 \mathrm{~mm}$ for the aSRR in its capacitive sections). (b) Frequency response (FEM simulation).

zero density at two independently engineered frequencies, and if placed in an otherwise all-pass acoustic duct, two notches in the frequency response can be observed. Here we show that the same unit cell can support simultaneous nearzero propagation at two frequencies if placed in a convenient host, such as the L section of the CLC filter used in the previous simulations. To that aim, a FEM simulation was carried out, with results shown in Figure 6(b), and it can be seen that two narrow passbands emerge in the stopband of the host. The dimensions used in the simulation were $R=5 \mathrm{~mm}$, $r_{1}=3.2 \mathrm{~mm}, r_{2}=3 \mathrm{~mm}, d_{1}=32 \mathrm{~mm}, d_{2}=4 \mathrm{~mm}$, $M_{1}=1 \mathrm{mg}$, and $M_{2}=0.88 \mathrm{mg}$, setting the two near-zero frequencies at $f_{1}=856 \mathrm{~Hz}$ and $f_{2}=1566 \mathrm{~Hz}$. At these two frequencies the effective density is equal to zero, as shown in the inset of Figure 6(b), which confirms the NZ character of acoustic wave propagation.

\section{Conclusions}

The paper proposes a novel approach to achieving density near-zero propagation of acoustic waves. A resonant unit cell previously employed for obtaining a double negative metamaterial is used to achieve propagation of acoustic waves with virtually no change in phase over physically long distances.

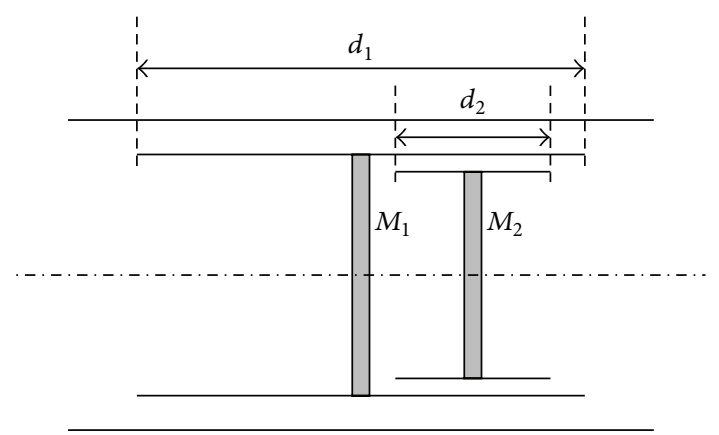

(a)

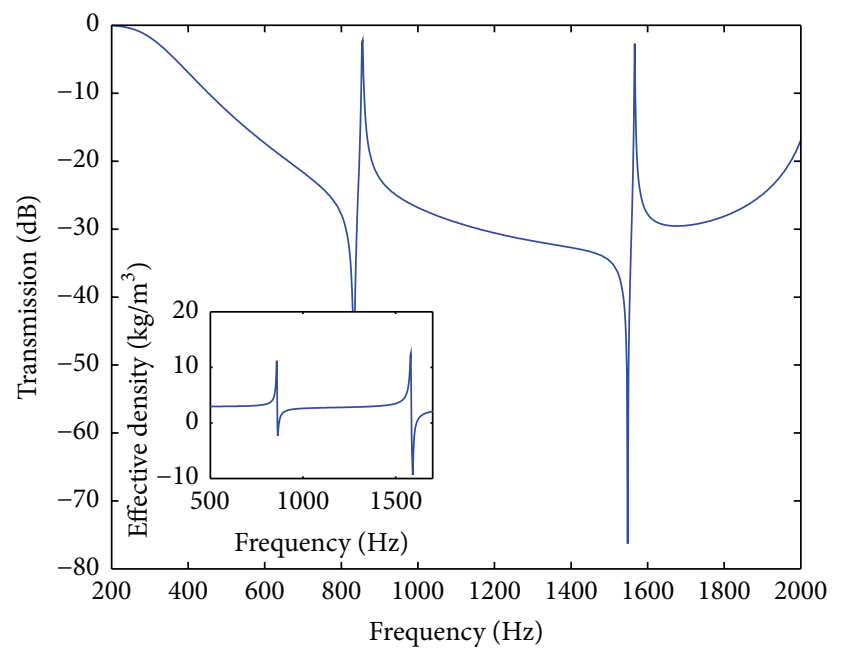

(b)

FIGURE 6: (a) Dual-band aSRR. (b) Near-zero wave propagation at two independently chosen frequencies, obtained by loading the inductive section of a CLC host with a dual-band aSRR (FEM simulation).

In this way the acoustic energy does not propagate but rather tunnels through a NZ metamaterial. Having in mind the wide range of application of NZ metamaterial structures, including energy tunneling, energy splitting, and perfect transmission through waveguides with sharp bends as well as various types of wave front manipulation, this research undoubtedly offers a valuable contribution in this field. Our future work will include the fabrication of the proposed unit cell and experimental verification of density near-zero propagation, as well as investigation of more complex structures based on the demonstrated phenomenon.

\section{Conflict of Interests}

The authors declare that there is no conflict of interests regarding the publication of this paper.

\section{Acknowledgment}

This research was supported in part by the European Commission (Grant no. PIRSES-GA-2009-247532). 


\section{References}

[1] Y. Ding, Z. Liu, C. Qiu, and J. Shi, "Metamaterial with simultaneously negative bulk modulus and mass density," Physical Review Letters, vol. 99, no. 9, Article ID 093904, 2007.

[2] Z. Yang, J. Mei, M. Yang, N. H. Chan, and P. Sheng, "Membranetype acoustic metamaterial with negative dynamic mass," Physical Review Letters, vol. 101, no. 20, Article ID 204301, 2008.

[3] S. H. Lee, C. M. Park, Y. M. Seo, Z. G. Wang, and C. K. Kim, "Acoustic metamaterial with negative modulus," Journal of Physics: Condensed Matter, vol. 21, no. 17, Article ID 175704, 2009.

[4] S. H. Lee, C. M. Park, Y. M. Seo, Z. G. Wang, and C. K. Kim, "Acoustic metamaterial with negative density," Physics Letters A, vol. 373, no. 48, pp. 4464-4469, 2009.

[5] S. H. Lee, C. M. Park, Y. M. Seo, Z. G. Wang, and C. K. Kim, "Composite acoustic medium with simultaneously negative density and modulus," Physical Review Letters, vol. 104, no. 5, Article ID 054301, 2010.

[6] F. Bongard, H. Lissek, and J. R. Mosig, "Acoustic transmission line metamaterial with negative/zero/positive refractive index," Physical Review B, vol. 82, no. 9, Article ID 094306, 2010.

[7] R. Fleury and A. Alù, "Extraordinary sound transmission through density-near-zero ultranarrow channels," Physical Review Letters, vol. 111, no. 5, Article ID 055501, 2013.

[8] N. Cselyuszka, M. Sečujski, and V. Crnojević-Bengin, "Novel negative mass density resonant metamaterial unit cell," Physics Letters A, vol. 379, no. 1-2, pp. 33-36, 2015.

[9] J. D. Baena, J. Bonache, F. Martín et al., "Equivalent-circuit models for split-ring resonators and complementary splitring resonators coupled to planar transmission lines," IEEE Transactions on Microwave Theory and Techniques, vol. 53, no. 4, pp. 1451-1460, 2005.

[10] S.-G. Mao, S.-L. Chen, and C.-W. Huang, "Effective electromagnetic parameters of novel distributed left-handed microstrip lines," IEEE Transactions on Microwave Theory and Techniques, vol. 53, no. 4, pp. 1515-1521, 2005.

[11] Z. Szabó, G.-H. Park, R. Hedge, and E.-P. Li, "A unique extraction of metamaterial parameters based on KramersKronig relationship," IEEE Transactions on Microwave Theory and Techniques, vol. 58, no. 10, pp. 2646-2653, 2010.

[12] A. H. Benade, "On the propagation of sound waves in a cylindrical conduit," Journal of the Acoustical Society of America, vol. 44, no. 2, pp. 616-623, 1968.

[13] D. H. Keefe, "Acoustical wave propagation in cylindrical ducts: transmission line parameter approximations for isothermal and nonisothermal boundary conditions," Journal of the Acoustical Society of America, vol. 75, no. 1, pp. 58-62, 1984.

[14] J. C. Zuercher, E. V. Carlson, and M. C. Killion, "Small acoustic tubes: new approximations including isothermal and viscous effects," The Journal of the Acoustical Society of America, vol. 83, no. 4, pp. 1653-1660, 1988. 

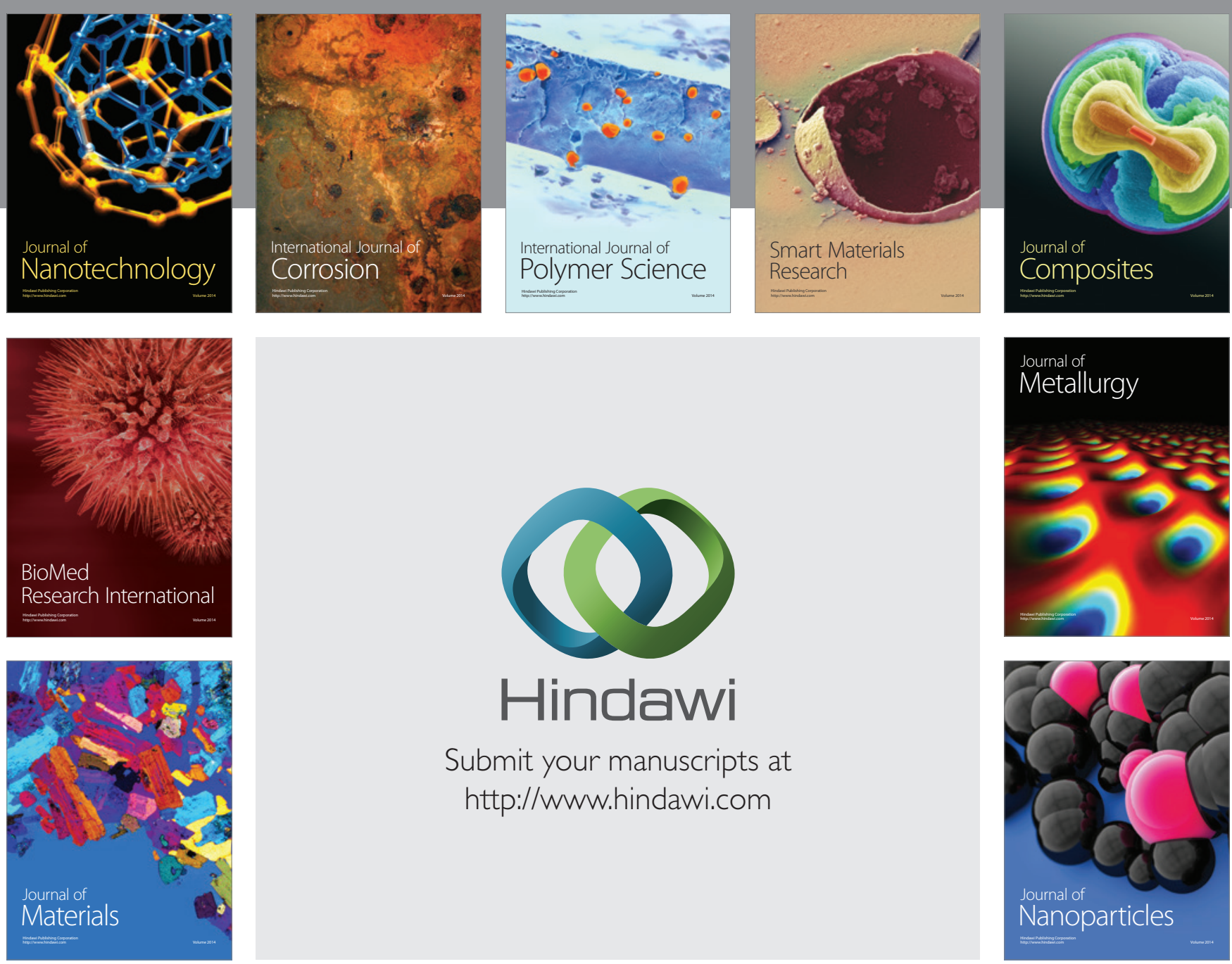

Submit your manuscripts at http://www.hindawi.com
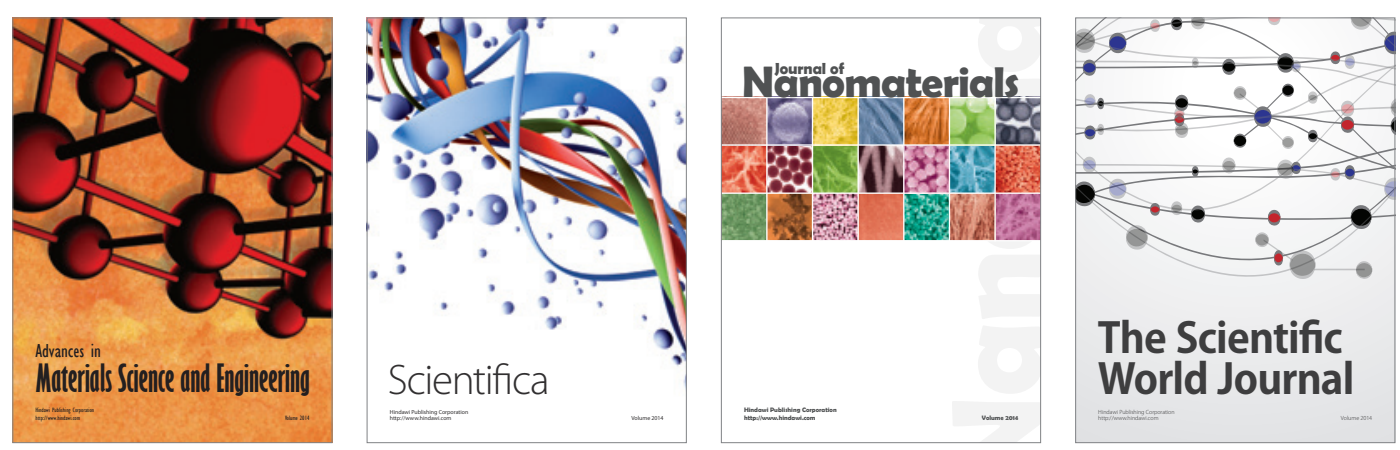

\section{The Scientific World Journal}
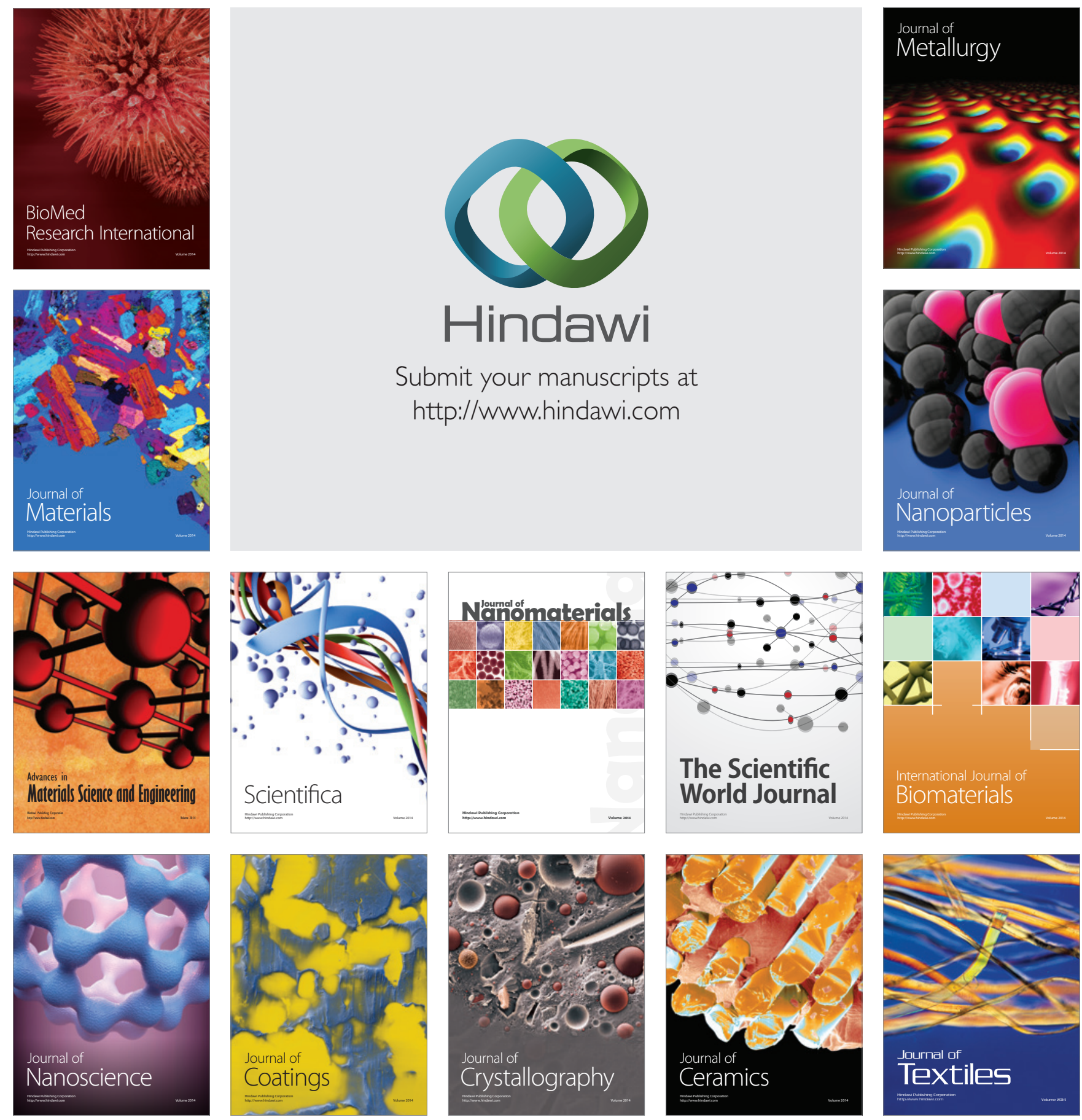